\title{
HETEROKARYON INCOMPATIBILITY AND VARIATION IN WILD POPULATIONS OF ASPERGILLUS NIDULANS
}

\author{
J. L. JINKS, C. E. CATEN, G. SIMCHEN and J. H. CROFT \\ Agricultural Research Council Unit of Biometrical Genetics, \\ Department of Geneties, University of Birmingham
}

Received I I.viii. 65

\section{INTRODUCTION}

THERE is a growing body of evidence which shows that in the homothallic Ascomycetes and imperfect fungi heterokaryons are formed only between a small proportion of the independent isolates of a species that can be obtained from the wild (Grindle, $1963 a$ and $b$; Jones, I 965 ; Caten, 1965; Caten and Jinks, 1966). The general features of this inability to form heterokaryons which have so far been established are: (i) The majority of pairwise combinations of the independent isolates fail to form heterokaryons, i.e. they are heterokaryon incompatible; (ii) The pattern of heterokaryon formation where it occurs is, with few exceptions, regular and predictable so that the isolates can be divided into heterokaryon compatibility groups; (iii) The isolates belonging to the same compatibility group are generally physiologically and morphologically very similar to each other and different from those of any other group (Grindle, $1963 b$ and unpublished Caten, 1965); (iv) The ability to form heterokaryons behaves as if it were under nuclear control, in a heterokaryon test (Jinks and Grindle, 1963).

These features indicate that heterokaryon compatibility depends upon the genotypic similarity of the paired homokaryotic isolates. This interpretation has been tested experimentally in Aspergillus nidulans with the results described below. At the same time we have investigated the consequences of heterokaryon incompatibility for the release of genetic variation at sexual reproduction in this fungus which, being homothallic, is potentially a complete inbreeder without the intervention of heterokaryosis (Pontecorvo, Roper, Henmons, Macdonald and Bufton, I953).

\section{ORIGIN OF STRAINS AND CROSSING PROGRAMME}

The strains used were all derived from seven independent wild isolates of Aspergillus nidulans. They will be referred to according to their number in the Birmingham collection of wild-type isolates, namely, 7, 9, 37, 40, 43, II 4 and 139 . The heterokaryon compatibility reactions of these isolates and other relevant information is summarised in table $\mathrm{I}$.

The following crosses were made.

(i) Crosses between derivatives of the same wild isolate:

Cross $\mathrm{I}-43 \times 43 \%$. 
(ii) Crosses between derivatives of different wild isolates belonging to the same compatibility group:

Cross $2-40 \times 43 y$, between isolates from the same locality.

Cross $3-43 y \times 139$, between isolates from different localities.

(iii) Crosses between derivatives of different wild isolates belonging to different compatibility groups:

Gross $4-7 y$ pro $_{4} \times 43$ an.
Cross $5-9 y$ bi $\times 43$ an.
Cross $6-37 y \times 114$.

TABLE I

The wild isolates and derivatives used in the experiments

\begin{tabular}{|c|c|c|l|}
\hline $\begin{array}{c}\text { Wild isolate } \\
\text { number }\end{array}$ & $\begin{array}{c}\text { Heterokaryon } \\
\text { compatibility group }\end{array}$ & $\begin{array}{c}\text { Mutant } \\
\text { derivatives }\end{array}$ & \multicolumn{1}{|c|}{$\begin{array}{c}\text { Locality of } \\
\text { isolation }\end{array}$} \\
\hline 7 & S & y, y pro & \\
9 & T & y bi, w & York \\
37 & Bombay \\
40 & E & y, & Birmingham \\
43 & Beamish, Co. Durham \\
114 & U & y, an & Beamish, Co. Durham \\
139 & Eembroke \\
& & $y$, & Aberystwyth \\
\hline
\end{tabular}

In every cross, the parents differed in that one had green and the other yellow $(y)$ asexual spores. Previous experience, confirmed in the present work, shows that the mutant allele $y$ has no effect on heterokaryon compatibility or, indeed, on any of the other characteristics of the strains that we are interested in. The parental strains used in crosses I, 2 and 3 produced heterokaryotic heads of asexual spores (mixed heads containing green and yellow spore chains) on contact. The wild type strains from which the parents used in crosses 4,5 and 6 were derived, did not, of course, form mixed heads when grown together. Furthermore, the auxotrophic derivatives used as parents of crosses 4 and 5 did not form mixed heads when grown together on a supplemented or complete medium that would support the growth of the parental strains. However, on a minimal medium these same combinations of parental strains readily produced mixed heads. In the firm belief that conditions favourable to the formation of mixed heads would also favour the production of crossed perithecia, all matings were made on a minimal medium at $25^{\circ} \mathrm{C}$. using a heavy suspension of the asexual spores of both parental strains as the inoculum. These attempts to overcome the heterokaryon incompatibility of the parental strains in crosses 4 and 5 may, in fact, have been unnecessary since crossed perithecia were obtained from cross 6 using two prototrophic parents belonging to different compatibility groups, which did not produce mixed heads during the mating. These observations, while not of immediate concern, raise important issues concerning the role 
of heterokaryosis in outcrossing in $A$. nidulans and the mechanism of heterokaryon incompatibility, which we shall return to in the discussion. For the present it is sufficient that all matings, after periods varying from two weeks to a month, yielded perithecia whose contents could only have arisen by outcrossing.

In seeking crossed perithecia, regions with high frequencies of mixed heads or failing this, regions where yellow and green heads of spores were well intermixed, were preferentially sampled. Segregation for the single gene difference, yellow versus green asexual spores, in the expected proportion was accepted as evidence that a perithecium

TABLE 2

The number of perithecia sampled and the number that were hybrid in origin in each of the six crosses

\begin{tabular}{|r|c|c|}
\hline Cross & Perithecia sampled & No. hybrid in origin \\
\hline (i) 1 & 12 & 2 \\
(ii) 2 & 29 & 4 \\
3 & 13 & 2 \\
(iii) & 17 & 3 \\
5 & 17 & 2 \\
6 & 12 & 2 \\
\hline
\end{tabular}

had a hybrid origin. This evidence was supplemented in crosses 4 and 5 by the segregation for the ability to grow on a minimal medium. The total number of perithecia sampled in each cross and the number that proved to have a hybrid origin are shown in table 2. On average I 5 per cent. of the perithecia were hybrid in origin and there is almost no variation in this respect between the six different matings. Since, however, the perithecia examined are highly selected samples one cannot safely infer anything from this similarity between matings.

\section{RESULTS}

\section{(i) Heterokaryon compatibility}

Samples of progeny from each of the six crosses were tested for their heterokaryon compatibility reaction with their own parental strains in the manner described by Grindle ( $1963 a$ and $b$ ). To carry out this test a number of conditions must be satisfied. First, the pair of strains to be tested must differ in the colour of their asexual spores. Since the progenies of each cross contain both yellow and green segregants, the former must be tested against a green spored (wild type) parental strain and the latter against a yellow or white spored derivative. Second, because the presence of auxotrophic mutations in a strain can modify its wild-type heterokaryon incompatibility reaction these tests have been carried out only between the prototrophic segregants of crosses 4 and 5 and the prototrophic strains from which the auxotrophic 
parents used in these crosses were derived. This requirement also makes it possible to carry out all tests on a Plunkett minimal medium (Plunkett, 1953) which in our experience is the best for detecting mixed heads. The results of the heterokaryon compatibility tests are summarised in table 3 .

On the basis of these results the six crosses fall into three groups corresponding with the three categories of cross made. In cross I, where the parental strains are not only heterokaryon compatible but also derivatives of the same wild isolate, all the progeny tested were compatible with the parents. Furthermore, in all combinations tested the frequency of mixed heads was uniformly as high as that obtained between the two parents themselves.

TABLE 3

The heterokaryon compatibility reactions of the sexual progenies of the six crosses when tested against their respective parental strains

\begin{tabular}{|c|c|c|c|c|c|c|c|}
\hline \multicolumn{2}{|l|}{ Cross type } & \multirow{2}{*}{ (i) } & \multicolumn{2}{|c|}{ (ii) } & \multicolumn{3}{|c|}{ (iii) } \\
\hline Cross number & . & & 2 & 3 & 4 & 5 & 6 \\
\hline $\begin{array}{l}\text { Sample tested } \\
\text { Compatible with both parents } \\
\text { Compatible with one parent only } \\
\text { Compatible with neither parent }\end{array}$ & $:$ & $\begin{array}{c}40 \\
40 \\
0 \\
0\end{array}$ & $\begin{array}{c}40 \\
40^{*} \\
0 \\
0\end{array}$ & $\begin{array}{c}40 \\
32^{*} \\
8 \\
0\end{array}$ & $\begin{array}{c}59 \\
0 \\
2^{*} \\
57\end{array}$ & $\begin{array}{c}44 \\
0 \\
2^{*} \\
4^{2}\end{array}$ & $\begin{array}{c}50 \\
0 \\
4^{*} \\
4^{6}\end{array}$ \\
\hline
\end{tabular}

* Although scored as compatible these are often less so than strains which belong to the same compatibility group (see text for details).

In the second category, crosses 2 and 3 where the parental strains were fully heterokaryon compatible but derived from independent wild isolates, the majority of the progeny (100 and 80 per cent., respectively) were compatible with at least one parent. However, even the progeny that were compatible were on average less so, as judged from the frequency of mixed heads, than were the two parental strains. This has been quantified by scoring the frequency of mixed heads on a scale from o to 6 , where 6 is the frequency of mixed heads produced by the parental combination. In table 4 the heterokaryon compatibilities of the progenies of crosses 2 and 3 are given on this scale. Their heterokaryon compatibilities are $8 \mathrm{r}$ and 63 per cent. respectively, of those shown by their parental strains and, of course, the progeny of cross $\mathrm{I}$.

An analysis of variance of the compatibility scores of the progenies of crosses 2 and 3 shows that individuals in the progeny of cross 3 differ significantly for this quantitative trait (table 4 ). Hence there is evidence that genes are segregating in this cross which effect the degree of compatibility. If this is so, we would expect a greater degree of compatibility between an offspring and one parent to coincide with 
a lesser degree of compatibility between the same offspring and the other parent. That is, we would expect a negative correlation between the scores obtained between the progeny and one parent and those obtained between the progeny and the other parent. This we in fact observed for the progeny of cross $3(r=-0.68, \mathrm{P}<0.00 \mathrm{I})$ but not as we might expect for that of cross 2.

While the progenies of crosses 2 and 3 are alike in showing a reduced compatibility, they differ consistently in that this reduction is qualitatively and quantitatively more marked in cross 3 . This coincides with the fact that the parents of cross 2 were isolated from different

TABLE 4

The quantitative aspects of the heterokaryon compatibility reactions of the progenies of crosses 2 and 3 when tested against their respective parental strains

\begin{tabular}{|c|c|c|}
\hline Cross & 2 & 3 \\
\hline $\begin{array}{c}\text { Mean compatibility } \\
\text { Variation in compatibility }\end{array}$ & $0.560(\mathrm{NS}) *$ & $3.56(\mathrm{P}<0.001)^{*}$ \\
\hline
\end{tabular}

* Tested against appropriate duplicate variance.

sites at the same locality while the parents of cross 3 were isolated from widely different localities (Grindle, $\mathrm{I}_{96}{ }^{6} b$ ). It could be, therefore, that geographical isolation is leading to genetic divergence even within the same compatibility groups.

The crosses in the third category $(4,5$ and 6$)$ are virtually identical in that their progenies show almost no heterokaryon compatibility with their own parental strains (table 3 ). Thus, only eight out of a total of 153 progeny tested from these three crosses produced any mixed heads in combination with their parents and only one of these achieved a compatibility score of 6 . Because of the small number of compatible combinations no analysis of the quantitative data has been attempted. It is nevertheless clear that in the crosses between heterokaryon incompatible parents there is a segregation for genes controlling compatibility and further that the genotypes of most of the resulting segregants are incompatible with those of their parents.

If we assume that for an offspring to be fully heterokaryon compatible with one of its parents it must carry identical alleles to that of the parent at a number of loci, then we can estimate this number as follows. At any one locus half the progeny will carry the allele present in one parent and half the allele present in the other parent. Thus if only one locus is involved all the progeny will be compatible with one or the other parent. Similarly, with two unlinked loci half the progeny will be fully compatible with one or the other parent. For $k$ unlinked loci, this proportion would be $2\left(\frac{1}{2}\right)^{k}$. For the three crosses, 4,5 and 6 where only one fully compatible offspring was recovered out of 153 , $k$ equals approximately eight. 
We can repeat this argument for progeny with reduced levels of heterokaryon compatibility which, presumably, have fewer identical alleles with the parents than progeny which are fully compatible. Thus to be compatible at all with their parents, the progeny of crosses 4,5 and 6 must have alleles in common at five loci, compared with eight for full compatibility. These estimates are of course subject to large sampling errors because of the small number of offspring involved. On the other hand, these are likely to be minimal estimates particularly if the controlling loci are linked.

TABLE 5

Analysis of mm. growth per day $\dagger$ in parental strains and their sexual progenies

\begin{tabular}{|c|c|c|c|c|c|c|}
\hline type & (i) & \multicolumn{2}{|l|}{ (ii) } & \multicolumn{3}{|c|}{ (iii) } \\
\hline Cross & I & 2 & 3 & 4 & 5 & 6 \\
\hline $\begin{array}{r}\text { Parents } \\
\text { mean } \\
\text { range }\end{array}$ & $\begin{array}{c}6 \cdot 0 \\
5 \cdot 9-6 \cdot 1\end{array}$ & $\begin{array}{c}6 \cdot 6 \\
6 \cdot 1-7 \cdot 2\end{array}$ & $\begin{array}{c}6 \cdot 0 \\
5 \cdot 7-6 \cdot 3\end{array}$ & $\begin{array}{c}5 \cdot 3 \\
5 \cdot 3 \cdot 5 \cdot 3\end{array}$ & $\begin{array}{c}6 \cdot 3 \\
5 \cdot 8-6 \cdot 7\end{array}$ & $\begin{array}{c}4 \cdot 5 \\
4 \cdot 2-4 \cdot 8\end{array}$ \\
\hline $\begin{array}{l}\text { Progeny } \\
\text { sample size } \\
\text { mean } \\
\text { range } \\
\text { Differences among } \\
\text { progeny }\end{array}$ & $\begin{array}{c}60 \\
5 \cdot 93 \\
5 \cdot 2 \cdot 6 \cdot 3 \\
P>0 \cdot 20\end{array}$ & $\begin{array}{c}9^{8} \\
6 \cdot 76 \\
6 \cdot 0 \cdot 7 \cdot 2 \\
P=0 \cdot 02-0 \cdot 05\end{array}$ & $\begin{array}{c}9^{8} \\
6 \cdot 57 \\
5 \cdot 5^{-8 \cdot I} \\
P<0 \cdot 001\end{array}$ & $\begin{array}{c}60 \\
4 \cdot 30 \\
3 \cdot 1-5 \cdot 9 \\
P<0 \cdot 001\end{array}$ & $\begin{array}{c}82 \\
5 \cdot 28 \\
3 \cdot 7 \cdot 6 \cdot 5 \\
P<0 \cdot 001\end{array}$ & $\begin{array}{c}99 \\
4 \cdot 04 \\
3 \cdot 1-4 \cdot 9 \\
P<0 \cdot 001\end{array}$ \\
\hline $\begin{array}{l}\text { Components of } \\
\text { variation } \cdot \\
\hat{\sigma}_{G}^{2} \cdot \\
\hat{\sigma}_{\mathbf{E}}^{2} \cdot \\
\text { Per cent. heritable } \\
\text { variation. }\end{array}$ & $\begin{array}{l}0.015 *(0.00) \\
0.104 \\
12.5^{*}(0.0)\end{array}$ & $\begin{array}{l}0.023 \\
0.111 \\
17.2\end{array}$ & $\begin{array}{l}0.098 \\
0.118 \\
45 \cdot 2\end{array}$ & $\begin{array}{l}0.327 \\
0.068 \\
82 \cdot 7\end{array}$ & $\begin{array}{l}0 \cdot 267 \\
0 \cdot 108\end{array}$ & $\begin{array}{l}0 \cdot 283 \\
0 \cdot 105 \\
72 \cdot 9\end{array}$ \\
\hline
\end{tabular}

$\dagger$ Since each cross was investigated independently direct comparisons of absolute growth rates between them should not be made.

* Since the mean square for difference among the progeny was not significant there is no evidence that $\hat{\sigma}_{\mathfrak{Q}}^{2}$ differs from zero.

\section{(ii) Variation in rate of growth}

The growth rates (that is, millimetres increase in diameter per day) of samples of the progenies of each of the six crosses were determined by growing them in duplicate on a minimal medium at $25^{\circ} \mathrm{C}$. for periods of up to eight days in a randomised block layout. Only prototrophic recombinants from crosses 4 and 5 were scored. The prototrophic strains from which the parents of crosses 4 and 5 were derived and the actual parents of the remaining crosses were also included in the experiment. The outcome of the various analyses of the growth rates obtained is summarised in table 5. Before, however, discussing the contents of this table a number of subsidiary analyses, not included in the table, will be considered. 
In the progeny of every cross there are green and yellow spored offspring. An analysis of variance of their respective growth rates showed that in every cross the spore colour difference was not associated with a significant difference in rate of growth. This confirms earlier experience. In the present experiment the implications are twofold. First the gene difference $Y, y$ has no pleiotropic effect on rate of growth and second, there is no evidence of linkage of loci controlling rate of growth to the yellow locus. In most cases progeny from two or more hybrid perithecia were examined. Again an analysis of variance gave no evidence that progeny from different perithecia differed in rate of growth. With these sources of variation ruled out, the items of interest reduce to those listed in table 5. For each cross, table 5 contains growth rates of the parents and their progeny, the results of a test of significance for variation in growth rate among the progeny, and an estimate of the contribution made by gene segregation $\left(\hat{\sigma}_{G}^{2}\right)$ and environmental differences $\left(\hat{\sigma}_{\mathrm{E}}^{2}\right)$ to the variation among the progeny (see Croft and Simchen, I 965 , for details).

Reference to table 5 shows that the crosses again fall into three categories which coincide with the type of relationship between the parental strains. In the cross between two derivations of the same wild isolate (cross $I$ ) there is no evidence of the segregation of genes controlling rate of growth and the parental and progeny means are identical. This cross is, of course, a control. We expect no segregation unless, as a result of spontaneous mutations, the two parental strains have diverged during their three years of independent culture in the laboratory. The fact that we have observed no segregation in this cross either for heterokaryon compatibility or for growth rate, suggests that we can safely ignore the possiblity of changes arising during laboratory subculture in interpreting the segregations observed in the other crosses. The remaining crosses, all of which are between different wild isolates, show significant evidence for the segregation of genes controlling rate of growth. There is a clear distinction, however, between crosses 2 and 3 on the one hand, and 4,5 and 6 on the other, both for the magnitude of this segregation and for the relationship between the growth rates of parents and progeny. Thus crosses 2 and 3, which are crosses within a compatibility group, show considerably less segregation of genes controlling growth rate than crosses 4,5 and 6 which are crosses between compatibility groups. Again the progeny of crosses 2 and 3 have growth rates that are as high or higher than those of their parents, while the progeny of crosses 4,5 and 6 have on average slower growth rates than those of their parents. These differences between the two types of cross (i.e. within and between heterokaryon compatibility groups) are shown in fig. I.

Cross 3 (between localities) once more shows a greater segregation than cross 2 (within locality). However, this greater segregation is still somewhat less than that observed for crosses 4,5 and 6 and is qualitatively quite different. In cross 3 the segregation is symmetrical 
around a mean that is higher than the parental mean, while the segregants in crosses 4,5 and 6 are asymetrically distributed around means that are lower than the corresponding parental means. In fact 8 o per cent. of the progeny of crosses 4,5 and 6 have growth rates which are slower than their own slower growing parent.

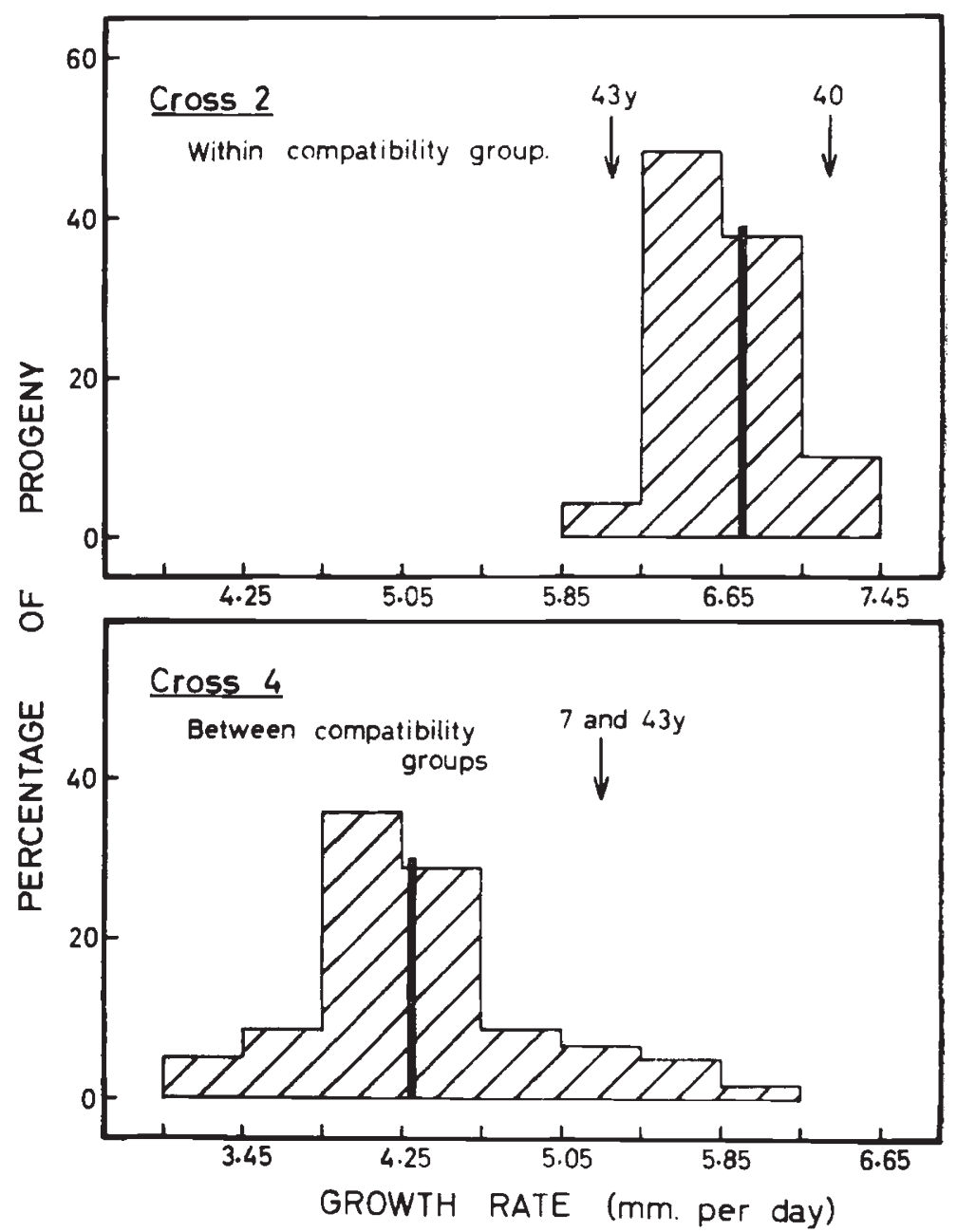

FIG. 1.-Frequency distributions of the progeny of crosses (i) between independent wild isolates belonging to the same heterokaryon compatibility group (cross 2) and (ii) between wild isolates belonging to different compatibility groups (cross 4 ). The arrows indicate the parental growth rates, and the bar the mean progeny growth rate. While the mean rate of growth of the progeny of cross 2 lies mid-way between the two parental growth rates, that for cross 4 is considerably less than the mid-parent value.

Since we are dealing here with haploid segregations we expect the parental and progeny means to be identical irrespective of the linkage relationships between the loci controlling growth rate, providing that there are no non-allelic interactions. If these are present then the difference between progeny and parental means is a direct estimate of the 
magnitude and sign of the interactions. Thus for crosses 2 and 3 there are some indications of non-allelic interactions of a positive kind, while for crosses 4,5 and 6 there are substantial non-allelic interactions of a negative kind. In the absence of non-allelic interactions, $\sigma_{G}^{2}=\mathrm{D}$, the additive genetic variance (Mather, I949), while in the presence of non-allelic interactions $\sigma_{G}^{2}=\mathrm{D}+\mathrm{I}_{d d}+\mathrm{I}_{d d d} \ldots$ where $\mathrm{I}_{d d}, \mathrm{I}_{d d d}$, etc., are the interactions between pairs, triplets, etc., of genes respectively.

\section{(iii) Variation in other characters}

Less detailed observations have been made on a variety of other characteristics of the progenies of the crosses including density of perithecia, morphology and background pigmentation. These observations, as far as they go, confirm the picture which has already emerged from the more detailed study of heterokaryon compatibility and rate of growth. Thus there is clear evidence of segregation for these characteristics in the progeny of the crosses between isolates belonging to different compatibility groups (crosses 4,5 and 6 ) and equally clear absence of segregation in the progeny of crosses between isolates belonging to the same compatibility group.

\section{DISCUSSION}

In $A$. nidulans (Grindle ${ }^{2} 963 b$ and unpublished) and in its asexual relatives $A$. terreus and $A$. versicolor (Caten, 1965) it is well established that while wild isolates with similar or identical phenotypes will readily form heterokaryons with one another, isolates with distinct phenotypes rarely, if ever, do so. It has also been established that within the British Isles there is no obvious geographical basis for the distribution of the different phenotypes encountered in these species. As a result, an isolate is just as likely to be able to form a heterokaryon with another isolate from a widely different location as it is with an isolate from the same location. Equally an isolate is just as likely to fail to form a heterokaryon with another isolate from the same location as it is to fail to form a heterokaryon with an isolate from a different location.

We have now established that the ability of pairs of isolates of $A$. nidulans to form heterokaryons is determined by the degree of similarity between their genomes. Isolates with similar or identical genotypes readily form heterokaryons while isolates with different genotypes either form heterokaryons less readily or not at all. Thus it appears that the heterokaryon compatibility test which we have used provides a direct measure of the relationship between the genotypes under test. The fact that the majority of pairs of wild isolates of $A$. nidulans are heterokaryon incompatible is therefore, indicative of the considerable genetic diversity which can be found in this species in nature. Our crosses between three heterokaryon incompatible pairs of wild isolates suggest that isolates which are incompatible differ at more than five loci. This number would be much greater if we had taken into 
consideration all the morphological and physiological characteristics which were independently segregating in the progenies. If the thirty or so heterokaryon incompatibility groups which have so far been recognised in a preliminary survey of the British Isles (Grindle, $1963 b$; and unpublished), each differ to this extent, then the heritable variation in this species is, by any standard, enormous.

The wild isolates that have diverged beyond the point where they can form heterokaryons with one another show indications of genetic isolation. When crossed they produce progenies in which the majority of the segregants are less vigorous than their poorer parent. Comparable results are commonly found when different, but related species, are intercrossed. Indeed the poorer viability and weakness of the progeny of such crosses is generally recognised as evidence that an internal barrier is acting as an isolating mechanism between the species (Mather, 1943, 1953, 1955; Stebbins, 1950).

In our earlier investigations on heterokaryon incompatibility we developed the argument that the incompatibility, by restricting heterokaryon formation to isolates with similar phenotypes, was responsible for the subdivision of the species into a number of groups between which there is virtually no gene exchange and within which gene exchange is possible but the genetic variation available for exchange is relatively trivial. Thus the genetic divergence was directly attributed to the operation of the incompatibility system. This, of course, is still one interpretation of the situation we have found in $A$. nidulans. However, it begs the question as to how the level of genetic diversity which is necessary to produce heterokaryon incompatibility itself arose. Furthermore, it assumes, what at the time seemed reasonable but seems less so now, that isolates which are heterokaryon incompatible would be unable to cross since in this homothallic fungus, heterokaryosis is an essential prerequisite for outcrossing to occur. Both points require further consideration in the light of our present findings.

It has been accepted for many years that heterokaryon formation is the first step in outcrossing, whether sexual or parasexual, in the homothallic and imperfect fungi (Pontecorvo, et al., r 953). We now find that pairs of isolates which we have classified as heterokaryon incompatible nevertheless yield some outcrossed progeny in the laboratory. We have classified as incompatible any pair of wild isolates which fail to produce heterokaryotic heads of spores, "mixed heads", when grown in mixed cultures under optimal laboratory conditions. Such a failure can have a variety of causes ranging from the inability of the isolates to anastomose to the failure of the heterokaryotic cells produced to successfully compete with the surrounding homokaryons from which they arose. The latter is not so much incompatibility as heterokaryotic disadvantage (Caten and Jinks, r966).

We can infer from the poor performance of the progeny of crosses between incompatible isolates that the genomes of such isolates are mutually unbalanced. Hence it follows that in a heterokaryotic 
association of such genomes this unbalance would lead to the inability of the heterokaryotic cells to compete with the homokaryotic balanced parents. Thus heterokaryon incompatibility in these isolates could have its origin in heterokaryotic disadvantage rather than failure to anastomose. This interpretation has the advantage that it is consistent with other properties of heterokaryon incompatible isolates. Thus there is no mechanism whereby the introduction of complementary auxotrophic mutants into incompatible isolates could force them to anastomose, when grown together on a minimal medium, if the prototrophic isolates are incapable of doing so. However, given that incompatible isolates always anastomose, and failure to produce heterokaryotic heads is a secondary effect arising from the lack of vigour of the heterokaryon, then it is easy to see how the selective advantage conferred on the heterokaryon by having complementary auxotrophic mutations in the incompatible isolates would lead to the successful establishment of the heterokaryon on a minimal medium.

All the available evidence from the study of naturally occurring and artificial heterokaryons of Ascomycetes and imperfect fungi suggests that their establishment, persistence and stability depends primarily on the greater vigour of the heterokaryotic association (Caten and Jinks, I966). In complete contrast, dikaryons whose independent properties can only be studied in Basidiomycetes, can be initiated and retained even where their rate of growth is less than either or both the monokaryotic components (Simchen and Jinks, I964). Clearly, vigour is not of primary importance in the establishment and persistence of the dikaryotic association of different genomes. The function of the dikaryon and hence the selective forces which have moulded the gene action during this stage in the life cycle, are quite different from that encountered in a homokaryon or a heterokaryon. If, therefore, we accept that heterokaryon incompatible isolates anastomose, it is possible that the initial heterokaryotic state which is unable to establish itself against vegetative competition, may nevertheless be capable of initiating a heterokaryotic dikaryon which successfully leads to the production of outcrossed sexual progeny.

On this interpretation heterokaryon incompatibility is not necessarily a bar to sexual outcrossing. Nevertheless, there is a bar to the free exchange of genes between incompatible isolates but it is imposed by an internal barrier or the imbalance in the resulting progeny (Mather, I943, I953, I 954). Thus, heterokaryon incompatibility now appears as a consequence of the genetic diversity and isolation within the species rather than its primary cause. On the other hand, the incompatibility, whether it is a primary or a secondary effect, restricts the potential vegetative plasticity of the fungus by preventing successful heterokaryon formation between the more diverse genotypes and it must have the same restrictive effect on the occurrence of the parasexual cycle.

Once the level of genetic diversity within a species has reached the 
point where the more diverse genotypes have an internal barrier to crossing, the diversity would be automatically maintained and even extended. The problem remains as to how this level was first achieved in A. nidulans. There are two possibilities. First, the genotypes which are classified as different compatibility groups may occupy different micro-ecological niches in which they are subject to different selective forces, even though they apparently have identical geographical distributions within the British Isles. Second, these genotypes may have diverged to the stage where they are genetically isolated at a time when the environment and geographical distribution was quite different from that encountered today. We cannot decide between these alternatives on the kind of evidence available at the present time. But, this aspect of the population genetics of $A$. nidulans along with the other problems we have raised about the meaning and role of heterokaryosis in the genetic system of this fungus, are currently being investigated further.

\section{SUMMARY}

The progeny of three kinds of crosses (i) between strains derived from the same wild isolate, (ii) between independent wild isolates belonging to the same heterokaryon compatibility group, and (iii) between independent wild isolates belonging to different compatibility groups, have been compared for a number of characteristics in order to investigate the mechanism and inheritance of heterokaryon incompatibility and its role in wild populations of Aspergillus nidulans.

The results show that while there is considerable heritable variation among wild isolates of $A$. nidulans, the variation is much greater among isolates belonging to different heterokaryon compatibility groups than among isolates belonging to the same group. Thus heterokaryon incompatibility is a result of the genetic diversity in this species. We estimate that for an offspring of an outcross to be compatible with one of its parents it must carry the alleles contributed by that parent at a minimum of five chromosomal loci.

The fact that progeny of crosses between heterokaryon incompatible strains are less vigorous than their parents suggests that "internal barriers" to outcrossing have evolved within this species. Thus isolates classified as belonging to different heterokaryon compatibility groups behave as sympatrically distributed but distinct species.

Preliminary results suggest that in this homothallic species heterokaryon incompatible isolates can be successfully crossed in the laboratory. Thus combinations of genomes that cannot establish themselves in heterokaryotic association in competition with their parental homokaryons can nevertheless successfully combine to initiate a dikaryon leading to the production of outcrossed sexual progeny. However, the poor performance of such progenies will presumably lead to their elimination in competition with the parental genotypes. Hence the 
genetic divergence which we have observed in the wild will be maintained.

Acknowledgments.--These investigations were carried out while one of us (C.E.C.) was in receipt of an Agricultural Research Council Research Studentship.

\section{REFERENCES}

CATEN, C. E. 1965. Asexual systems of variation in fungi. Ph.D. thesis, University of Birmingham Library.

CATEN, C. E., AND JINKS, J. L. 1966. Heterokaryosis: its significance in wild homothallic Ascomycetes and fungi imperfecti. Trans. Brit. Mycol. Soc.

CROPT, J. H., AND SIMCHEN, G. 1965. Natural variation among monokaryons of Collybia velutipes. Amer. Naturalist, 99, 45 I-462.

GRINDLE, M. 1963a. Heterokaryon compatibility of unrelated strains in the Aspergillus nidulans group. Heredity, 18, 191-204.

GRINDLE, M. $1963 b$. Heterokaryon compatibility of closely related wild isolates of Aspergillus nidulans. Heredity, 18, 397-405.

JINKS, J. L., AND GRINDLE, M. 1963. The genetical basis of heterokaryon incompatibility in Aspergillus nidulans. Heredity, $18,407-413$.

JONES, D. A. 1965. Heterokaryon compatibility in the Aspergillus glaucus Link. group. Heredity, 20, 49-56.

MATheR, X. 1943. Polygenic inheritance and natural selection. Biol. Revs., 18, $32-64$.

MATHER, K. 1949. Biometrical Genetics. Methuen, London.

MATHER, K. 1953. The genetical structure of populations. Symp. Soc. Expt. Biol., $7,274-289$.

MATHER, K. 1955. Polymorphism as an outcome of disruptive selection. Evolution, 9, $52-6 \mathrm{I}$.

PLUNKETT, B. E. 1953. Nutritional and other aspects of fruit body formation in pure culture of Collybia velutipes. Ann. Bot. N.S., 17, 193-21 7 .

PONTECORVO, G., ROPER, J. A., HEMMONS, L. M., MACDONALD, R. D., AND BUFTON, A. W. J. 1953. The genetics of Aspergillus nidulans. Adv. Genet., 5, 141-238.

SIMChEN, G., AND JiNKs, J. L. 1964. The determination of dikaryotic growth rate in the Basidiomycete Schizophyllum commune: a biometrical analysis. Heredity, 19, 629-649.

stebrins, G. L. 1950. Variation and Evolution in Plants. Columbia Univ. Press, New York. 